\title{
Discovering Convolutive Speech Phones Using Sparseness and Non-negativity
}

\author{
Paul D. O'Grady ${ }^{1}$ and Barak A. Pearlmutter ${ }^{2}$ \\ ${ }^{1}$ Complex \& Adaptive Systems Laboratory, University College Dublin, \\ Belfield, Dublin 4, Ireland \\ ${ }^{2}$ Hamilton Institute, National University of Ireland Maynooth, \\ Co. Kildare, Ireland \\ paul.d.ogrady@ucd.ie, barak@cs.nuim.ie
}

\begin{abstract}
Discovering a representation that allows auditory data to be parsimoniously represented is useful for many machine learning and signal processing tasks. Such a representation can be constructed by Non-negative Matrix Factorisation (NMF), which is a method for finding parts-based representations of non-negative data. Here, we present a convolutive NMF algorithm that includes a sparseness constraint on the activations and has multiplicative updates. In combination with a spectral magnitude transform of speech, this method extracts speech phones that exhibit sparse activation patterns, which we use in a supervised separation scheme for monophonic mixtures.
\end{abstract}

\section{Introduction}

A preliminary step in many data analysis tasks is to find a suitable representation of the data. Typically, methods exploit the latent structure in the data. For example, ICA [1] reduces the redundancy of the data by projecting the data onto its independent components, which can be discovered by maximising a statistical measure such as independence or non-Gaussianity.

Non-Negative Matrix Factorisation (NMF) approximately decomposes a nonnegative matrix $\mathbf{V}$ into a product of two non-negative matrices $\mathbf{W}$ and $\mathbf{H}[2,3]$. NMF is a parts-based approach that does not make a statistical assumption about the data. Instead, it assumes that for the domain at hand, negative numbers are physically meaningless. Data that contains negative components, for example audio, must be transformed into a non-negative form before NMF can be applied. Here, we use a magnitude spectrogram. Spectrograms have been used in audio analysis for many years and in combination with NMF have been applied to a variety of problems such as sound separation [4] and automatic transcription of music [5].

In this paper, we combine a previous convolutive extension of NMF 4] with a sparseness constraint on $\mathbf{H}$, and present an algorithm that has multiplicative updates. This paper is structured as follows: We overview convolutive NMF in Section 2 and present sparse convolutive NMF in Section 3 . In Section 4 we apply sparse convolutive NMF to speech spectrograms, and extract phones that 
have sparse activation patterns. We use these phones in a supervised separation scheme for monophonic mixtures, and demonstrate the superior separation performance achieved over those extracted by convolutive NMF in Section 5 .

\section{Convolutive NMF}

NMF 3 is a linear non-negative approximate factorisation, and is formulated as follows. Given a non-negative matrix $\mathbf{V} \in \mathbb{R}^{\geq 0, M \times T}$ the goal is to approximate $\mathbf{V}$ as a product of two non-negative matrices $\mathbf{W} \in \mathbb{R} \geq 0, M \times R$ (basis) and $\mathbf{H} \in$ $\mathbb{R}^{\geq 0, R \times T}$ (activations), $\mathbf{V} \approx \mathbf{W H}$, where $R \leq M$, such that the reconstruction error is minimised. For our purposes we require a convolutive basis, such a model has previously been used to extend NMF [4, which we review in this section.

For conventional NMF each object is described by its spectrum and corresponding activation in time, while for convolutive NMF each object has a sequence of successive spectra and corresponding activation pattern across time. The conventional NMF model is extended to the convolutive case:

$$
\mathbf{V} \approx \sum_{t=0}^{T_{o}-1} \mathbf{W}_{t} \stackrel{t \rightarrow}{\mathbf{H}} \quad v_{i k} \approx \sum_{t=0}^{T_{o}-1} \sum_{j=1}^{R} w_{i j t}\left(\stackrel{t \rightarrow}{h_{j k}}\right)
$$

where $T_{o}$ is the length of each spectrum sequence and the $j$-th column of $\mathbf{W}_{t}$ describes the spectrum of the $j$-th object $t$ time steps after the object has begun. The function $(\cdot)$ denotes a column shift operator that moves its argument $i$ places to the right; as each column is shifted off to the right the leftmost columns are zero filled. Conversely, the $\overleftarrow{(}^{i} \cdot$ operator shifts columns off to the left, with zero filling on the right. We use the beta divergence, which is a parameterisable divergence, as the reconstruction objective,

$$
D_{\mathrm{BD}}(\mathbf{V} \| \boldsymbol{\Lambda}, \beta)=\sum_{i k}\left(v_{i k} \frac{v_{i k}^{\beta-1}-[\boldsymbol{\Lambda}]_{i k}{ }^{\beta-1}}{\beta(\beta-1)}+[\boldsymbol{\Lambda}]_{i k}^{\beta-1} \frac{[\boldsymbol{\Lambda}]_{i k}-v_{i k}}{\beta}\right),
$$

where $\beta$ controls reconstruction penalty and $\boldsymbol{\Lambda}$ is the current estimate of $\mathbf{V}$, $\boldsymbol{\Lambda}=\sum_{t=0}^{T_{o}-1} \mathbf{W}_{t} \stackrel{t \rightarrow}{\mathbf{H}}$. The choice of the $\beta$ parameter depends on the statistical distribution of the data, and requires prior knowledge, see [6, Chapter 3]. For $\beta=2$, Squared Euclidean Distance is obtained; for $\beta \rightarrow 1$, the divergence tends to the Kullback-Leibler Divergence; and for $\beta \rightarrow 0$, it tends to ItakuraSaito Divergence. It is evident that Eq. 1 can be viewed as a summation of $T_{o}$ conventional NMF operations. Consequently, as opposed to updating two matrices ( $\mathbf{W}$ and $\mathbf{H}$ ) as in conventional $\mathrm{NMF}, T_{o}+1$ matrices require an update $\left(\mathbf{W}_{0}, \ldots, \mathbf{W}_{T_{o}-1}\right.$ and $\left.\mathbf{H}\right)$. The resultant convolutive NMF update equations are

$$
w_{i j t} \leftarrow w_{i j t} \frac{\sum_{k=1}^{T}\left(v_{i k} /[\boldsymbol{\Lambda}]_{i k}^{2-\beta}\right) h_{j k}^{t \rightarrow}}{\sum_{k=1}^{T}[\boldsymbol{\Lambda}]_{i k}^{\beta-1} \stackrel{h \rightarrow}{t \rightarrow}_{h_{j k}}^{t}}, \quad h_{j k} \leftarrow h_{j k} \frac{\sum_{i=1}^{M} w_{i j t}\left(v_{i k} /\left[\overleftarrow{\Lambda}_{i k}^{2-\beta}\right)\right.}{\sum_{i=1}^{M} w_{i j t}[\overleftarrow{\boldsymbol{\Lambda}}]_{i k}^{\beta-1}},
$$


where $\mathbf{H}$ is updated to the average result of its updates for all $t$. When $T_{o}=1$ this reduces to conventional NMF.

\section{Sparse Convolutive NMF}

Combining our reconstruction objective (Eq. 2) with a sparseness constraint on $\mathbf{H}$ results in the following objective function:

$$
G(\mathbf{V} \| \boldsymbol{\Lambda}, \mathbf{H}, \beta)=D_{\mathrm{BD}}(\mathbf{V} \| \boldsymbol{\Lambda}, \beta)+\lambda \sum_{j k} h_{j k},
$$

where the left term of the objective function corresponds to convolutive NMF, and the right term is an additional constraint on $\mathbf{H}$ that enforces sparsity by minimising the $L_{1}$-norm of its elements. The parameter $\lambda$ controls the trade off between sparseness and accurate reconstruction.

\subsection{Basis Normalisation}

The objective of Eq. 4 creates a new problem: The right term is a strictly increasing function of the absolute value of its argument, so it is possible that the objective can be decreased by scaling $w_{i j t}$ up and $\mathbf{H}$ down $\left(w_{i j t} \mapsto \alpha w_{i j t}\right.$ and $\mathbf{H} \mapsto(1 / \alpha) \mathbf{H}$, with $\alpha>1)$. This situation does not alter the left term in the objective function, but will cause the right term to decrease, resulting in the elements of $w_{i j t}$ growing without bound and $\mathbf{H}$ tending toward zero. Consequently, the solution arrived at by the optimisation algorithm is not influenced by the sparseness constraint.

To avoid the scaling misbehaviour of Eq. 4 another constraint is needed; by normalising the convolutive bases we can control the scale of the elements in $w_{i j t}$ and $\mathbf{H}$. Here, normalisation is performed for each object matrix, $\mathbf{W}_{j}$, by rescaling it to the unit $L_{2}$-norm, $\overline{\mathbf{W}}_{j}=\frac{\mathbf{W}_{j}}{\left\|\mathbf{W}_{j}\right\|}, j=1, \ldots, R$, where the matrix $\mathbf{W}_{j}$ is constructed from the $j$-th column of $w_{i j t}$ at each time step, $t=0,1, \ldots, T_{o}-1$.

\subsection{Multiplicative Updates}

Multiplicative updates can be obtained by including the normalisation requirement in the objective. Previously, this has been achieved for conventional NMF using the Squared Euclidean Distance reconstruction objective [7]. Here, we present the multiplicative updates for a convolutive NMF algorithm utilising beta divergence. Our new reconstruction objective is a modification of Eq. 2 where each object, $\mathbf{W}_{j}$, is normalised, $\overline{\mathbf{W}}_{j}$, resulting in the following generative model: $\boldsymbol{\Delta}=\sum_{t=0}^{T_{o}-1} \sum_{j=1}^{R} \overline{\mathbf{w}}_{j t}\left(\mathbf{h}_{j}\right)$. By substituting $\boldsymbol{\Lambda}$ for $\boldsymbol{\Delta}$ in Eq. 4 we obtain [6. Chapter 4] the following multiplicative update rules for $\mathbf{H}$ and $\mathbf{W}$ :

$$
h_{j k} \leftarrow h_{j k} \frac{\sum_{i=1}^{M} \bar{w}_{i j t}\left(v_{i k} /\left[\boldsymbol{\Delta}_{i k}^{2-\beta}\right)\right.}{\sum_{i=1}^{M} \bar{w}_{i j t}\left[\overleftarrow{\boldsymbol{\Delta}}^{t}\right]_{i k}^{\beta-1}+\lambda},
$$



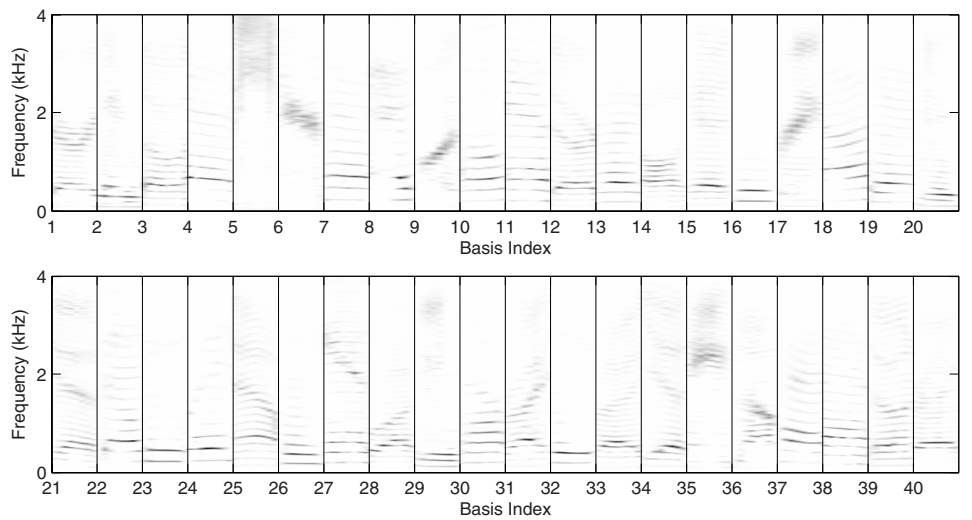

Fig. 1. A collection of 40 phone-like basis functions for a mixture of a male (DMT0) and female speaker (SMA0) taken from the TIMIT speech database

$$
w_{i j t} \leftarrow w_{i j t} \frac{\sum_{k=1}^{T} \stackrel{t \rightarrow}{h}_{j k}\left[\left(v_{i k} /[\boldsymbol{\Delta}]_{i k}^{2-\beta}\right)+\bar{w}_{i j t}\left(\bar{w}_{i j t}[\boldsymbol{\Delta}]_{i k}^{\beta-1}\right)\right]}{\sum_{k=1}^{T}{ }^{t \rightarrow} h_{j k}\left[[\boldsymbol{\Delta}]_{i k}^{\beta-1}+\bar{w}_{i j t}\left(\bar{w}_{i j t}\left(v_{i k} /[\boldsymbol{\Delta}]_{i k}^{2-\beta}\right)\right)\right]}
$$

\section{Sparse Convolutive NMF Applied to Speech Spectra}

We apply sparse convolutive NMF to speech, and present a learned basis for the sparse representation of speech using the TIMIT database. Recently, such work has been presented for convolutive NMF [8].

\subsection{Discovering a Phone-Like Basis}

To illustrate the differences between the phones extracted by convolutive NMF and sparse convolutive NMF we perform the following experiment for both algorithms: We take around 15 seconds of speech from a male (DMTO) and female (SMA0) speaker to create a contiguous mixture. The data is normalised to unit variance, down-sampled from $16 \mathrm{kHz}$ to $8 \mathrm{kHz}$ and a magnitude spectrogram of the data is constructed. We use a FFT frame size of 512, a frame overlap of 384 and a Hamming window to reduce the presence of sidelobes. We extract 40 bases, $R=40$, with a temporal extent of 0.176 seconds, $T_{o}=8$, and run convolutive NMF (with $\beta=1$ ) for 200 iterations. The extracted bases are presented in Figure 1. The experiment is repeated for sparse convolutive NMF with $\lambda=15$, and the corresponding bases are presented in Figure 2

For convolutive NMF, it is evident that the extracted bases correspond to speech phones. The verification of which, can be achieved by listening to an audible reconstruction. Most of the phones represent harmonic series with differing pitch inflections, while a smaller subset of phones contain wideband components that correspond to consonant sounds. It is evident for the harmonic phones that 

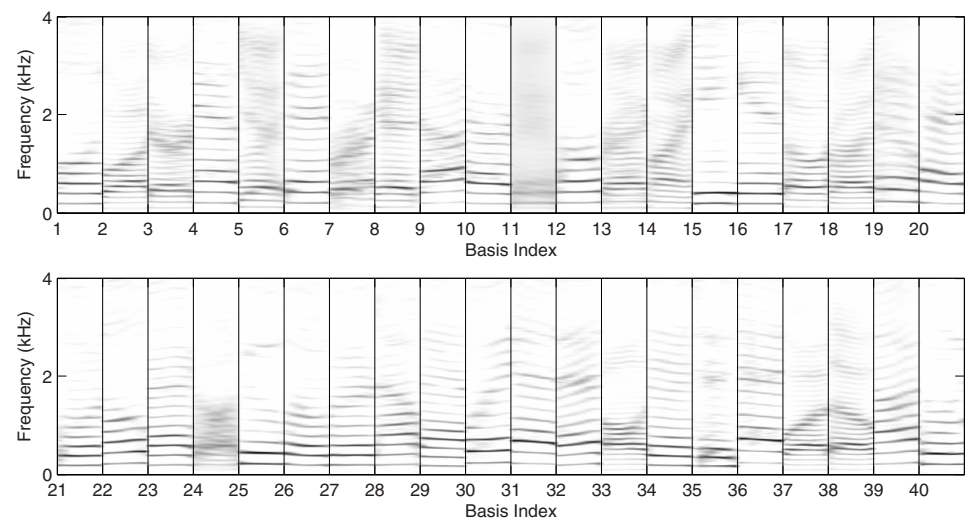

Fig. 2. A collection of 40 phone-like basis functions for a a mixture of a male (DMT0) and female speaker (SMAO) taken from the TIMIT speech database. The basis is extracted using Spare Convolutive NMF with $\lambda=15$.

some bases have harmonics that are spaced much closer together, which is indicative of a lower pitched male voice, while others are farther apart, indicating a higher pitched female voice. Therefore, it is evident that the extracted phones correspond to either the male or female speaker, which indicates that the timbral characteristics of the male and female speaker are sufficiently different, such that phones that are representative of both cannot be extracted.

By placing a sparseness constraint on the activations of the basis functions, we specify that the expressive power of each basis be extended such that it is capable of representing phones parsimoniously, much like an over-complete dictionary. The result is that the extracted phones exhibit a structure that is rich in phonetic content, where harmonics at higher frequencies have a much greater intensity than seen in the phones extracted by convolutive NMF. Analysis of the male and female sparse phone set reveals another important difference between the two speakers. In addition to difference in harmonic spacing, it is evident that the structure of the male phones are of a more complex nature, where changes over time are much more varied than for the female phone set.

\section{Supervised Method for the Separation of Speakers}

As illustrated in our previous experiments, the structure of the bases that are extracted from the speech spectrogram are uniquely dependent on the speaker (given the same algorithm parameters). In the context of speech separation, it is not unreasonable to expect that the bases extracted for a specific speaker adequately characterise the speaker, such that they can be used to discriminate them from other speakers. For a monophonic mixture where a number of speakers are summed together, it is possible to separate the speakers in the mixture by constructing an individual magnitude spectrogram from each speaker, using the 
phones specific to that speaker. More formally, we use the following procedure for the separation of a known male and female speaker from a monophonic mixture:

1. Obtain training data for the male, $s_{m}(t)$, and female, $s_{f}(t)$, speaker, create a magnitude spectrogram for both, and extract corresponding phone sets, $\mathbf{W}_{t}^{m}$ and $\mathbf{W}_{t}^{f}$, using sparse convolutive NMF.

2. Construct a combined basis set $\mathbf{W}_{t}^{m f}=\left[\mathbf{W}_{t}^{m} \mid \mathbf{W}_{t}^{f}\right]$, which results in a basis that is twice as big as $R$.

3. Take a mixture that is composed of two unknown sentences voiced by our selected speakers, and create a magnitude spectrogram of the mixture. Fit the mixture to $\mathbf{W}_{t}^{m f}$ by performing sparse convolutive NMF with $\mathbf{W}_{t}$ fixed to $\mathbf{W}_{t}^{m f}$, and learn only the associated activations $\mathbf{H}$.

4. Partition $\mathbf{H}$ such that the activations are split into male, $\mathbf{H}^{m}$, and female, $\mathbf{H}^{f}$, parts that correspond to their associated bases, $\mathbf{H}=\left[\mathbf{H}^{m} \mid \mathbf{H}^{f}\right]^{\top}$.

5. Construct a magnitude spectrogram for both speakers, using their respective bases and activations: $\mathbf{S}^{m}=\sum_{t=0}^{T_{o}-1} \mathbf{W}_{t}^{m} \mathbf{H}^{m}$ and $\mathbf{S}^{f}=\sum_{t=0}^{T_{o}-1} \mathbf{W}_{t}^{f} \mathbf{H}^{f}$.

6. Use the phase information from the mixture to create an audible reconstruction for both speakers.

This procedure may also be used for convolutive NMF, and can be generalised for more than two speakers, and speakers of the same gender.

\subsection{Separation Experiments}

Here, we compare the separation performance of convolutive NMF and sparse convolutive NMF. For an extensive study of the relationship between parameter selection and separation performance for convolutive NMF, see [8].

We select three male (ABCO, BJVO, DWMO) and three female (EXMO, KLHO, REHO) speakers from the TIMIT database, and create a training set for each that includes all but one sentence voiced by that speaker. We artificially generate a monophonic mixture by summing the remaining sentences for a selected malefemale pair, for a total of nine mixtures. Each sentence pair is normalised to unit variance, down-sampled from $16 \mathrm{kHz}$ to $8 \mathrm{kHz}$, and summed together. A magnitude spectrogram of each mixture is constructed using an FFT frame size of 512, a frame overlap of 256 and a Hamming window.

The separation performance for both algorithms is evaluated for each mixture over a selection of values for $R\left(R=\left\{\begin{array}{lll}40 & 80 & 140220\end{array}\right)\right.$. For both algorithms the temporal extent of each phone is set to 0.224 seconds $\left(T_{o}=6\right)$, the number of iterations is $150, \beta$ is set to 1 and each experiment is repeated for 10 Monte Carlo runs. For convolutive NMF, a total of 24 speaker phone sets are extracted and used in $360(9 \times 4 \times 10)$ separation experiments. For sparse convolutive NMF

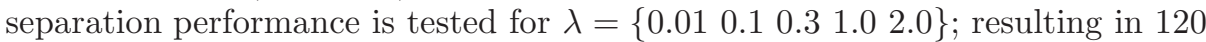
$(6 \times 4 \times 5)$ speaker phone sets and $1800(9 \times 4 \times 5 \times 10)$ separation experiments.

For the purposes of ease of comparison with existing separation methods, we evaluate the separation performance of both algorithms using the Sourceto-Distortion Ratio (SDR) measure provided by the BSS_EVAL toolbox 9]; SDR 


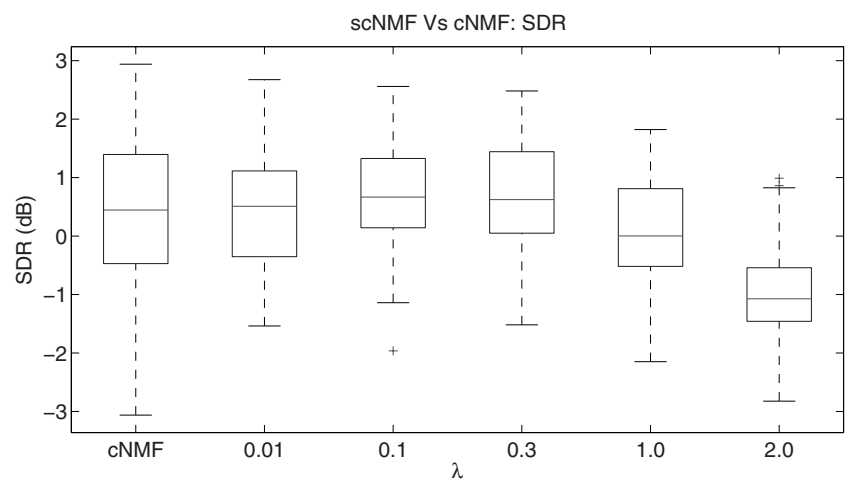

Fig. 3. A comparison of the SDR results obtained by convolutive and sparse convolutive NMF: Box plots are used to illustrate the performance results, where each box represents the median and the interquartile range of the results. It is evident that for $\lambda=0.1$, a better spread of results is obtained, indicating that sparse convolutive NMF achieves superior overall performance.

indicates overall separation performance and is expressed in $\mathrm{dB}$, with higher performance values indicating better quality estimates. An extensive investigation utilising all measures provided by the toolbox is presented in [6, Chapter 4].

\subsection{Separation Performance}

We statistically analyse the performance of convolutive NMF and sparse convolutive NMF by collating the results from all experiments and presenting the results using box plots: Each box presents information about the median and the statistical dispersion of the results. The top and bottom of each box represents the upper and lower quartiles, while the length between them is the interquartile range; the whiskers represent the extent of the rest of the data, and outliers are represented by + . Box plots for SDR are presented in Figure 3 ,

The SDR results indicate that for $\lambda=\{0.1,0.3\}$, the median performance obtained $(0.66 \mathrm{~dB}, 0.62 \mathrm{~dB})$ exceeds convolutive NMF $(0.44 \mathrm{~dB})$, for our given algorithm parameters. It is also evident that a better spread of results is produced for sparse convolutive NMF; demonstrating that when $\lambda$ is chosen appropriately, sparse convolutive NMF achieves superior overall performance. However, audible reconstructions reveal that convolutive NMF is more resilient to artifacts; this may reflect the fact that each sparse phone set exhibits phones that are rich in features, which may manifest as artifacts in the resultant source estimates. It is also evident that the performance of the sparse convolutive algorithm degrades significantly for large $\lambda$, so much so, that it renders the results useless, for our data this is especially evident for $\lambda>1$. 


\section{Conclusion}

In this paper, we presented a sparse convolutive NMF algorithm with multiplicative updates, which effectively discovers a sparse parts-based convolutive representation for non-negative data. This method extends the convolutive NMF objective by including a sparseness constraint on the activation patterns, enabling the discovery of over-complete representations. Furthermore, we demonstrated the superiority of sparse convolutive NMF over convolutive NMF, when applied to a supervised monophonic speech separation task.

\section{Acknowledgements}

Supported by Higher Education Authority of Ireland (An tÚdarás Um ArdOideachas), and Science Foundation Ireland grant 00/PI.1/C067.

\section{References}

[1] Comon, P.: Independent component analysis: A new concept. Signal Processing 36, 287-314 (1994)

[2] Paatero, P., Tapper, U.: Positive matrix factorization: A nonnegative factor model with optimal utilization of error estimates of data values. Environmetrics 5, 111-126 (1994)

[3] Lee, D.D., Seung, H.S.: Algorithms for non-negative matrix factorization. In: Adv. in Neu. Info. Proc. Sys. 13, pp. 556-562. MIT Press, Cambridge (2001), URL citeseer.ist.psu.edu/lee00algorithms.html

[4] Smaragdis, P.: Non-negative matrix factor deconvolution; extraction of multiple sound sources from monophonic inputs. In: Puntonet, C.G., Prieto, A.G. (eds.) ICA 2004. LNCS, vol. 3195, pp. 494-499. Springer, Heidelberg (2004)

[5] Abdallah, S.A., Plumbley, M.D.: Polyphonic transcription by non-negative sparse coding of power spectra. In: Proceedings of the 5th International Conference on Music Information Retrieval (ISMIR 2004), pp. 318-325 (2004)

[6] O'Grady, P.D.: Sparse Separation of Under-Determined Speech Mixtures. PhD thesis, National University of Ireland Maynooth (2007), URL

http://ee.ucd.ie/ pogrady/ogrady2007_phd.pdf

[7] Eggert, J., Körner, E.: Sparse coding and NMF. In: IEEE International Joint Conference on Neural Networks, Proceedings, July 2004, vol. 4, pp. 2529-2533. IEEE, Los Alamitos (2004)

[8] Smaragdis, P.: Convolutive speech bases and their application to supervised speech separation. IEEE Transaction on Audio, Speech and Language Processing (2007)

[9] Févotte, C., Gribonval, R., Vincent, E.: BSS_EVAL toolbox user guide. Technical Report 1706, IRISA (2005) 